\title{
Sachregister zu Band 64
}

Die fett gedruckten Zahlen bezeichnen Eigenarbeiten. Bb. = Buchbesprechung. A.

Ablatio retinae, doppelseitige - bei Schwangerschaftsniere 328.

- Operation der - 77.Aderhaut s. Chorioidea.Akinesie bei Augenoperationen

79.Akkommodationskrampf, ein-

sei tiger - Ill.

Albinos, Tätowierung der Binde-haut rn.it Goldchlorid bei -- 77.

Alkoholinj ektionen in das Ganglion Gasseri bei Trigeminusneur-algie 330.

orbitale 320.

retrobulbäre 83. Ammoniakverätzung des Auges

120.

Anaesthetica 325.

Aneurysma der Arteria cerebralis anterior mit Koiripression der Sehnerven und des Chiasma 335.

- $\quad$ der Carotis interna 315.Angioma sclerae 400.Angiospasmus retinalis, hervorgerufen durch metallische

Fremdkörperim Angeninnern 406. Aniridia partialis und Polykorie

197. Aniridie, Extractio cataractae bei

angeborener -- 354. AntiviГus, Besredkasches 189. Apicosanbehandlung bei Iritis

rheumatica 188. Argyrose, Berufs- - der Cornea

393-

Arteria centralis retinae, Em-bolie der -- 78.

- $\quad$ Embolie der - mit Ausgang vonden Tonsillen 104.

Arteriosklerose, $\Lambda$ lyopie bei -

112. Aspergillus, Granulom der Orbita

durch - 202. Asterol 185. Astigmatismus, Bestimmung des

- $\quad$ durch die Schattenprobe mitZylindergläsern 91, 92.

- $\quad$ Kobaltlampe zur Bestimmung des

- $\quad 160$.

- $\quad$ Wund-A. naeh Kataraktextrak-tion 81.

Atropininj ektionen, Behandlung der Hypotonie mit subkonjunkti-valen"- 337 .

Augapfel s. Bulbus. Augenbrauen, plastische Neubil-

dung von -- 74. Augendruck s. Druck. Augenhintergrund, Blutungen

des - bei Neugeborenen 174.

- $\quad$ Faltenbildung am - 164.Augenhintergrundsphotogra-

phien, stereoskopische 196. Augenlider s. Lider. Augenmuskelstörungen, seltene

Fälle zentraler -- no. Augenoperationen 72 (Bericht). Augenverletzungen 338.

- direkte Verletzungen des Sehnerven 400.

- durch Klettenstacheln 357. 
B.

Bakterioskopische Prophylaxe bei Kataraktoperationen 80.

Berufsargyrose der Cornea 393.

Besredkasches Antivirus 189.

Betäubung 72.

Bewegungsstörungen des Auges, Messung und Darstellung der - 100.

Bindehaut s. Conjunctiva.

Biprismalgläser, Wirkung der--109.

Bißwunde, geheilte - der Conjunctiva, Sklera, Chorioidea und Retina mit Erhaltung des Augapfels und seiner Funktion 390.

Blaulicht-Augenbestrahlungs-lampe 168.

Blepharospasmus, Operation des

- 75-Blindenwesen 122, 340. Blutdruckmessung in den intra-

okularen Arterien 101. Blutungen, expulsive - nach Star-

operationen 80. Blutversorgung des Auges 113. I Brillengläser, Breite der prak-

tischen Dezentralisierungsmög-

lichkeit von - 402. í Bulbusverletzungen, perforie-

rende - durch feinste Draht-

stückchen 190.

C. ! Carotisinterna, Aneurysma der --

$3 \frac{1}{8} 5$

Sachregister zu Band $64 . \quad 409$

$\mathrm{C}$ h i a s m a, Kompression des - durch

ein Aneurysma der Arteria cere-

bralis anterior 335. Chininvergif tung 112. Chirurgie des Augapfels und seiner

Adnexe 347 (Bb.). Cholesterinausstreuung in der

vorderen Augenkammer als Ur-.

sache von Glaukom bei über-

reifem Altersstar 46. Cholesterinkristalle in alten

Netzhautblutungen 393. Chorioidealarterien, pulsierende

158. Chorioidealkarzinom, metastati-

sches 395. Chorioide alsarkom, frühzeitige

extraokulare Metastase eines -

196. Chorioidealtumor, Operation

eines -77 .

- $\quad$ Pigmentation und Entstehungdes - 323.

Conjunctiva, multiple Abszesse

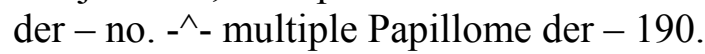

- $\quad$ Plastik nach Verätzungder - 75.Conjunctivitisgonorrhoica, sel-

tene Hornhautkomplikationen bei

- 149.

Cornea, Berufsargyrose der -393 .

- $\quad$ üermoid der -176.

eigenartige zentrale Veränderung der - im Senium 366.

Entwicklung der -324 .

Entwicklungsgeschichte der -293. 
-naht nach Staroperation 197. -narben, Aufhellung von - in.

-oberfläche, Darstellung der -und ihrer Veränderungen im Re-ílexbild 404.

Operationen an der - 76, 77.

- $\quad$ operative Ablösung flächenhafterVerwachsungen zwischen Iris und

- $\quad 182$.

Pigmentablagerungen auf der Hinterf läche der - in der Ehrlich-schen Linie 198.

- $\quad$ Pruning der Empfindliehkeit der

- $\quad$ gegen Druckausübung am Lideals diagnostisches Hilfsmittel 127.

- Reflexbilder der - in,

- seltene Komplikationen der - bei Conjunctivitis gonorrhoica 149.

- $\quad$-Spiegelin Medizin und Kunst no.Corodenin 118.

Corpus vitreum, Ansaugen des - 78 .

- $\quad$ Blutung des - nach Schädel-trauma 40.

Corpus vitreum, metastatisches Karzinom des - 191.

senile Trübungdes - und Lupen-spiegeluntersuchung 95.

Vorfall des - nach Diszissionen 81.

Cysticercus im Glaskörper, Ansaugen des - 78.

I).

Dermoid der Cornea 176. Descemetsche Membran, Abrei-

Bung der - nach Staroperation

81. üiaphanoskopie, retrobulbäre 96. Diplopie, monokuläre 203.

- postoperative 75.Dislocatio bulbi 397.Diszission, Komplikationen der -

81. Doppelbilder, die diagnostische

Verwertung der - 109. Drehnystagmus, optischer 328.

- $\quad$ Vorrichtung zur Auslösung desoptischen - 101.

Drehtrommel, optische 328.

üruck, intraokularer, Abhängig-keit des - von der Wasserstofí-ionenkonzentration des Blutes 43.

- $\quad$ Steigerung des - durch Muskel-aktionen 79.

Driisenbildung, exzessive symme-trische - in der hinteren Pol-gegend 389.

E.

Ektropium senile. Operation des

- 398.

EUiotsche Trepanation 83. Enophthalmus traumaticus 78,

197. Entropium, Operation des - 74. Enukleation, Stumpfbildung nach

321. Ephetonin 117. Epitarsus 324. Ergotamin 317. Erwärmbarkeit der lichtbrechen-

den Teile des Auges nach Be-

strahlung durch einen leuchten-

den Körper 143. Exenteratio bulbi 396.

- $\quad$ Meningitis nach - 78.Exophthalmus 195.

F.

Faltenbildung am Augenhinter-

grund 164. Farbenblindheit, apoplektiform

entstandene totale - 395. Farbenperimetrie, Bedeutung der

- $\quad$ beim Glaukom 184.

4io

Sachregister zu Band 64. 
Farbensinn, individuelle Verschie-

denheit des -; no. Farbensinnprüfung 94, 95. Färbungen, vitale - am mensch-

lichen Auge 1. Feuermal und Glaukom 396. Fibrolysin, Einträufelung von -

326. Fremdkörper 78, 79, 98, no, 324.

Lokalisation der - 336 .

Mydriasis und Angiospasmus reti-nalis, hervorgerufen durch metal-lische - im Augeninnern 406.

Schrotkorn im Auge 394.

GL

Ganglion Gasseri, Alkoholinjek-

tionen in das - bei Trigeminus-

neuralgie 330. Gasbazillus, Panophthalmitis

durch den Fränkelschen - 175. Glasbläserstar als Gewerbekrank-

heit 338. Glaskörper s. Corpus vitreum. Glaukom, basale Iridektomie bei -

130.

Bedeutung der Farbenperimetrie beim - 184.

Cholesterinausstreuung in der vor-deren Augenkammer als Ursache von - bei überreifen

Altersstar 40.

Radiumbehandlung bei hämor-rhagischem - 156.

und Feuermal 396.

Wirkungsweise der medikamen-tösen Behandlung des-117, 201.

Glaukomoperationen 82, 83,183.

Glaukosan 310, 312.

Gliom, Röntgenbestrahlung des -

193-

- und Trauma 121.

Glioma retinae, doppelseitiges

334-

- $\quad$ Zellbefunde bei - 193.

Gol-dchlorid, Tätowierung der Hornhaut mit - 76, 77, 119, 326.

Granulom der Orbita durch Asper-gillus 202.

Grenzstrahlen, Wirkung der Buckyschen - auf das Auge 316.

$\mathrm{H}$.

Haarnaht geschlitzter Tränenkanäl-

chen 352. Halb-Elliot 373. Helladaptation, eine Lichtfläche

zur Normierung der -95 . Herpes corneae 175. Herpes tonsurans 112. Heterochromie,

Sympathikus- -

105.

Hirntumoren 331.

- $\quad$ Art der Einwirkung der Röntgen-strahlen bei - 33 .

Hornhaut s. Cornea. Hypotonie des Auges 337. Hypophysistumor, Bestrahlung eines - 103.

I.

Ikterus, Sehpurpurbildung bei -

09. Interf erometrie 102. Iridektomie 82.

basale - bei Glaukom 130 . 
schmale - bei Staroperationen 198.

Iridocyclitis tuberculosa 329. Iridoenkleisis antiglaukoma-

tosa 83. Iris, Lymphwege der -320.

Membranbildungen auf der Vor-derfläche der - 278.

metastatisches Karzinom der - 191.

-nekrose, akute primäre -227.

operative Ablösung flächenhafter Verwachsungen zwischen Cornea und - 182.

Pigmentringlinie auf der - nach. perforierender Verletzung 106.

Rubeosis iridis diabetica no.

-tuberkulose 329.

Verfärbung der - nach Radium-bestrahlung 103.

Iritis, Apicosanbehandlung bei rheumatischer - 188. - und Neuritis optica bei Weilscher Krankheit 222.

J.

Jo dip in als Kontrastmittel in der Radioskopie der Tränenwege $98>$ Jodisan 319.

K.

Kalkverätzung 192.

Kammerwasser, Wasserstof $\mathrm{f}$ ionen-konzentration des -335 .

Kaninchenaugensarkom, Meta-stasen bei -272.

Kaninchensarkom, Transplantation von - auf Kaninchenaugen 259.

Kanthotomie 79.

Karzinom, metastatisches - der Iris und des Corpus ciliare 191.

Katarakt, familiäre juvenile 323.

- Glasbläserstar als Gewerbekrank-heit 338.

Sachregister zu Band 64.

$3 / 8 \mathrm{II}$

Katarakta, senilis, Cholesterinaus-streuung in der vorderen Augen-kammer als Ursache von

Glau-kom bei überreifer - 46 .

Kataraktoperationen79, 80, Ill,

"5.

bei angeborener Aniridie 354.

bei Altersstar 118, 119.

bei Luxation der Linse in die Vorderkammer 400.

Hornhautnaht nach - 197.

schmale Iridektomie bei - 198. Keratitis photoelectrica 403.

sympathica 170. Keratokonus, Atiologie des - Ill. - Behandlung des - 317 .

Kontaktglas bei - 103. Keratoplastik 77. Kinderlähmung, spinale, iso-

lierte Lähmung des Obliquus inferior bei - 127 Klettenstacheln, Augenverletzun-

gen durch - 357. Knochenbrüchigkeit und blaue

Sklera 391. Kobaltlampe zur Bestimmung des

Astigmatismus 160. Kokain, Ersatzpräparate des - 72. Kokain-Adrenalintamponade

der Nase zur Behandlung der

retrobulbären Neuritis 22. Kolmatage 397. Kolobom der Netzhaut 65. Kontaktglas bei

Keratokonus 103. 
- $\quad$ bei Macula corneae 313.Kontrastfarben, Ursachen der -

322. Kristalle, sphärolithische - in der

Linse 394. Kryptophthalmus congenitus

240. Kupfersplitter im Auge 324.

L.

Lagophthalmus, Operation des

paralytischen - 74. Laktation, Neuritis optica während

der -102 . Lidfixierung bei bulbuseröffnen-

den Operationen 79. Lidkarzinom 400. Lidoperationen 74, 75. Lidplastik, kombiniert mit Tarsus-

Transplantation 398. Linse, sphärolithische Kristalle in

der - 394. Linsenluxation, Extraktion der

in die Vorderkammer luxierten

Linse 400.

- $\quad$ in die Vorderkammer 330.Linsentrübungen bei Neugebore-

nen 174.

Lochbildung, metazystische - mit

guter zentraler Señschärfe 160. ' Lues der Orbita 330. Lues cerebrospinalis, Blässe des

Sehnerven ohne Sehstörung bei -

204. Lufteinblasung, Behandlung der

tabischen Sehnervenatrophie mit

- $\quad$ 107, 377.Lupenspiegeluntersuchung und

senile Glaskörpertrübung 95. Lymphräume, Sichtbarkeit peri-

vaskulärer - der Netzhaut 109. Lymphwege der Iris 320.

M.

Macula, beiderseitige Veränderun-

gen der - bei drei Geschwistern

388. Macula corneae, Kontaktgläser

bei - 313. Magnet, Ansatz zum Haabschen -

190. Magnetextraktion 78. Melanome, sklerale Durchleuch-

tung von - 323. Membranbildungen auf der Iris-

vorderfläche 278. Meningitis nach Exenteration des

Augapfels 78. Mikroorganismen, diepathogenen

- des Auges 123 (Bb.).Mikrophthalmus 200.

- myopischer 173.Miliaraneurysmenretinitis 314.Musculus obliquus inferior, iso-

lierte Lähmung des - bei spinaler Kinderlähmung 327.

- $\quad$ Myektomie des - 75.Muskelvorlagerung, Resultate

der - 399. Myastheniagravispseudopara-

lytica, Augenmuskelstörungen

bei - 112, 316. Mydriasis, hervorgerufen durch

metallische Fremdkörper im Au-

geninnern 406. Myopie bei Arteriosklerose 112.

Genese der -113 .

N. Nachstaroperationen 81. Nahpunkt, Apparat zur genauen

Bestimmung des - 392. Nasale Veränderungen am Auge

108. Nekrolysin 73. Nervusoculomotorius, Lähmung

des - nach Zahnextraktion 192. 
Sachregister zu Band 64.

Nervus opticus-Atrophie, ein Ver- I such, den Verlauf der tabischen

- $\quad-$ zu beeinflussen 50 .

-, Behandlung der tabischen - mit Lufteinblasung T07, 377.

- $\quad$ hereditäre - - bei Vater undSohn 165.

- $\quad$ - Kopfröntgenogramme von -392 .

Blässe des - ohne Sehstörung bei Cerebrospinallues 204.

direkte Verletzungen des -400.

endonasale Eingriffe bei Erkran-kung des - 78 .

Kompression des - durch ein Aneurysma der Arteria cerebralis anterior 335.

-Scheiden-Trepanation bei Stau-ungspapille 103.

Netzhaut s. Retina. Neugeborene, Augenbefunde bei

- 174-

Neuritis optica bei Weilscher

Krankheit 222.

- $\quad$ während der Laktation 102.

- retrobulbaris 106, 158, 162,

177. 178.

- $\quad$ und ihre Behandlung mit Kokain-Adrenalintamponade der Nase 22.

Nystagmograph 99.

0 .

Ophthalmia nodosa durch Rau-penhaare 393.

Ophthalmoplegie, chronische progressive 136.

- $\quad$ komplette beiderseitige - beiMyasthenie 112 .

Opticus s. Nervus opticus.

Optochin-Iontophoresei72,2ot.

Or bit a, operative Beseitigung der leeren - in.

-Orbitalphlegmone, Thrombophlebitis der kontralateralen Orbita bei Otitis media 109.

Otitismedia, Thrombophlebitis der kontralateralen Orbita nach - 109.

Orbitaltumoren 112.

P.

Pannus trachomatosus, Schleim-haut-Transplantation nach Denig gegen - 398.

Panophthalmiti s durch Fränkel-schen Gasbazillus 175.

Papillome, multiple - der Conjunctiva 190.

Paralyse, progressive, Beteili-gung der Sehrinde an dem histo-pathologischen Prozeß der - 403.

Pathologie und pathologische

Anatomie des Auges 125 “(Bb.). Pemphigus beider Augen 191. Perimetrie 96.

- $\quad$ Bedeutung der Farben - - beimGlaukom 184.

- $\quad$ Überblicks-P. 187.Phlyktäne, Ätiologie der - 188.Piezometer 168.Pigmentablagerungen auf der

Hinterfläche der Cornea in der Ehrlichschen Linie 198.

Pigmentzerfall, akuter der Netzhaut infolge von Septojodintoxi-kation 320. 
Polykorie und Aniridia partialis 197.

Prismen-Ref raktometer 167.

Protargolgranulat 407.

Psikain 72.

Ptosis, Operation der -75.

Pupillarmembran, persistierende 174.

Pupillendistanzmesser 392.

Pupillometer, optisches 98.

ß.

Radiumbehandlung bei hämor-rhagischem Glaukom T56.

- $\quad$ Irisverfärbung nach - 103.Raupenhaare, Ophthalmia nodosa

durch - 393. Reflektogene Veränderungen

am Auge 108. Reflexbilder der Hornhaut in. Reflexphotographie 161. Retina, akuter Pigmentzerf

all der

- $\quad$ infolge von Septojodintoxika-tion 320.

- $\quad$ abnormer Verlauf eines Gefäí3esder - und Kolobombildung der

$-\quad \operatorname{ti} \Lambda$.

-blutungen, Cholesterinkristallein alten -- 393-

- $\quad$-gefäße, Dreiteilung der- 112.

- $\quad$ scheibenartige Entartung der --mitte und Tuberkulose 163.

- $\quad$ Sichtbarkeit perivaskulärerLymphräume der - 109.

Retinitis, Miliaraneurysmen-

$3 \mathrm{H} \cdot$

- $\quad$ mit massiger Exsudation 405.Retinitis pigmentosa, Einteilung

der - in erblich verschiedene

Formen 325. Röntgenaufnahme, skelettfreie

98. Röntgenstrahlen, Augenschädi-

gungen durch -- 161.

- $\quad$ Art der Einwirkung der - beiHirntumoren 33.

Rubeosis iridis diabetica no.

Sachregister zu Band 64. 413

S. Sanochrysin 322. Sarkom, Metastasen von Kanin-chenaugen- - 272.

multiple hämorrhagische der Au-genlider und der Bindehaut 197.

Transplantation von Kaninchen-- auf Kaninchenaugen 25\$).

Schädeltrauma, Glaskörperblu-

tung nach -40 . Scharfer L.öffel bei Hornhaut-

erkrankungen 76. Schei nbewegung, optische 324. Schemata zur Einzeichnung von

Doppelbildern und Augenstellun-

gen 100. Schleimhaut- Transplantation

nach Denig gegen Pannus tracho-

matosus 398. Sdimerzstillung 72. Schrotkorn im Auge 394. Sehnerv s. Nervus opticus.

Schwangerschaftsniere, doppel-

seitige Netzhautablösung bei -

328. S eh p rob en 94.

Sehpurpurbildung bei Ikterus 6Í). Sehrinde, Beteiligung der - an

dem histopathologischen Prozeß 
der progressiven Paralyse 403. Sehschwachenschule 319. Sehvermögen, Prüfung des -94 .

Senium, eigenartige zentrale Horn-

hautveränderung im -366 . Septoj odintoxikation, akuter

Pigmentzerfall der Netzhaut in-

folge von - 320. Siderosis bulbi 157. Skiaskopie $91 \mathrm{ff}$. Skier a, Angiom der - 400.

- $\quad$ blaue - und Knochenbrüchigkeit39i.

- $\quad$ Operation des Staphyloms der -77-

Sklerale Durchleuchtung von

Melanomen 323. Sklerometer 98. Somnifen 72.

Spaltlampenmikroskopie 96. Sphärolithische Kristallein der

Linse 394. Staphylom der Sklera, Operation

des - 77. Staphyloma verum maculare,

beiderseitiges 390. Stauungspapille, Optikusschei-

dentrepanation bei - 103. Strabismus, Operation des -75 .

- $\quad$ scheinbares Auswärtsschielen ei-nes Auges infolge abnorm großenAbstandes von Fovea und Pupille349.

Strabismus, Therapie und Pro-

gnose des - 109. Stumpfbildung nach Enukleation

321. Sympathikusheterochromie --

105. Sympathische Entzündung und

sympathische Reizung 116.

T.

Tarsustransplantation, kombi-

niert mit Dieffenbach-Arltscher

Lidplastik 398. Tätowierung der Hornhaut mit

Goldchlorid 76, 77. Thérapeutique chirurgicale

ophthalmologique 126 (Bb.). Thermokauter bei Keratitis 76. Thrombophlebitis der kontralateralen Orbita bei Otitis media 109. Tonometer, das neue X.-T. von

Schiötz 327. Tonometrie 99. Tonsillen, Ausgang einer Embolie

der Zentralarterie von den - 104. Tophus syphiliticus der Tränen-

sackgegend 109. Trachom in Argentinien 107. Tränendrüse, Exstirpation der

palpebralen - 74. Tränenkanälchen, P2rkrankung

der -190 .

- Haarnaht geschlitzter - 352.Tränensack, Sarkom des - 324.Tränensackexstirpation

73. Tränensackkanalspritze zurMes-

sung des Druckes 99. Traumatische Neurose 121. Trepanation bei Ulcus serpens 76. Trichiasis, Behandlung der - 74. Trigeminusneuralgie, Alkoholin-

jektionen in das Ganglion Gas-

seri bei - 330. Tuberkulin, Rolle des - im ge-

sunden und kranken Organismus

205. Tuberkulose des Auges 204 (Bb.).

- $\quad$ und scheibenförmige Entartungder Netzhautmitte 163.

Tumordiagnostik, Bedeutung der Interferometrie für die - 102 .

Tunica vasculosa lentis, seltene Reste der -169 .

Tupferfadenin der Vorderkammer nach Staroperationen 80.

Tutokain 72. 
U.

Uberblicksperimetrie 187. Ulcus rodens corneae 331.

Zeitschrift für AugenheUkunde. Bd. 64. Heft 6.

414

Sachregister zu Band 64.

Ulcus serpens, Trepanation der

Hornhaut bei - 76. Unfallkunde 120, 338. Unf all verhütung bei Augenver-

letzungen 318. Untersuchungsm ethoden 89

(Bericht). Uviollicht 97. Uvioltherapie 200.

Verätzungen des Auges 120, 170,

192. Yerkupferung des Auges 393. Versicherungskunde 120, 338. Yertikalmotoren, Vorrichtung zur Bestimmung der Lähmung

der -321 . Vitale Färbungen am mensch-

lichen Auge 1. - des vorderen Augenabschnittes 97. Vivokoll zur Blutstillung bei Trä-

nensackexstirpation 73. Vorderkammer, Entwicklung der

$-324-$

W.

Wasserstoffionenkonzentra-

tion des Blutes, Abhängigkeit des

intraokularen Druckes von der -

48. - des Kammerwassers 335. Weilsche Krankheit, Iritis und

Neuritis optica bei - 222. Wimpern, plastische Neubildung

von -74 . Wundastigmatismus nach Kata-

raktextraktion 81 . Wundsprengungen nach Star-

operationen 80.

$\mathrm{X}$.

Xanthomatosis bulbi, Frühsta-dium von - 195.

Z.

Zahnextraktion, Okulomotorius-

lähmung nach - 192. Zonulaschwund 319. Zügelnaht bei Kataraktoperation

79-Zyklodialyse 82. Zylinderskiaskopie 91, 92.

Namenregister zu Banð 64.

Alsberg 165. Amersbach 108. Apin 99. Arkin 74. Ascher 72, 82. 109. Asmus 76, 321. Avizonis 240. Axenfeld 79, 180, 182,

194.

B. B a c h m a n n 74. Bartels 319, 32T, 327,

328, 329. Behr 81, 344. Best 80.

Bielschowsky 75, 109. Birch-Hirschfeld 80,

125-Birnbacher 227. Blank 388. v. Blaskovics 197,

198, 397. 398, 399,

400.

Bliedung 98. Bl.och 192. Boente 394. Borinski 112, 168. Braun 109. Braunstein 74, 75. Butler 203.

C.

Caspary 81. Clausen 162, 163, 164, 
167, 172, 174, 175. Comberg 79, 114, 127,

394-Cords 319. Cramer 170. v. Csapody 396, 397, 399, $4^{\circ} 2 \cdot$ Czellitzer 113.

$1)$.

Davids 78. Deutsch 156. Dinger 102.

Drescher 95. Du verger 126.

E.

Elschnig, A. no, 118,

II9 .

Elschnig, H. H. 78, 80,

99, 109, 112. Endres 43. Engelbrecht 73, 164,

167, 171. Erggelet 161 .

v. Fazakas 200. Fecht 331. Feigenberg 114. Fejer 197. Filatow 77. Fischer, F. 293. Fischer, F. P. in, 161, 